\title{
Guillaume Blanc, Élise Demeulenaere et Wolf Feuerbahn (dir.), 2017, Humanités environnementales, enquêtes et contre-enquêtes, Paris, Publications de la Sorbonne, 350 pages.
}

\section{Claude Kergomard}

\section{(2) OpenEdition}

\section{Journals}

Édition électronique

URL : http://journals.openedition.org/developpementdurable/12095

DOI : 10.4000/developpementdurable.12095

ISSN : 1772-9971

Éditeur

Association DD\&T

Référence électronique

Claude Kergomard, « Guillaume Blanc, Élise Demeulenaere et Wolf Feuerbahn (dir.), 2017, Humanités environnementales, enquêtes et contre-enquêtes, Paris, Publications de la Sorbonne, 350 pages. », Développement durable et territoires [En ligne], Vol. 9, n¹ | Mars 2018, mis en ligne le 30 mars 2018, consulté le 24 septembre 2020. URL : http://journals.openedition.org/developpementdurable/12095 DOI : https://doi.org/10.4000/developpementdurable.12095

Ce document a été généré automatiquement le 24 septembre 2020.

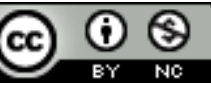

Développement Durable et Territoires est mis à disposition selon les termes de la licence Creative Commons Attribution - Pas d'Utilisation Commerciale 4.0 International. 
Guillaume Blanc, Élise Demeulenaere et Wolf Feuerbahn

(dir.), 2017, Humanités environnementales, enquêtes et contreenquêtes, Paris, Publications de la Sorbonne, 350 pages.

Claude Kergomard 


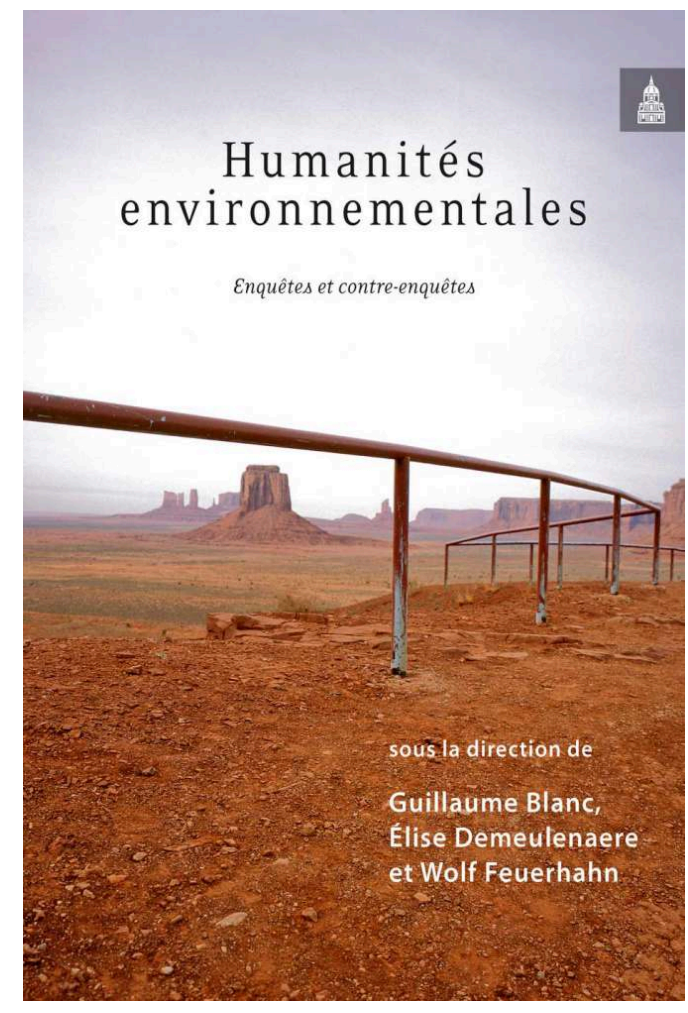

1 Issu d'un séminaire de master associant le Museum d'Histoire Naturelle et l'École des Hautes Études en Sciences Sociales, cet ouvrage collectif se propose d'analyser l'impact sur les différentes sciences humaines et sociales des enjeux écologiques et climatiques contemporains, sous leurs formes globales ou locales, et l'émergence au sein de celles-ci de courants qui, prenant l'environnement pour objet principal, tendraient à converger dans les « humanités environnementales ", une méta-discipline fédérant des approches nées soit au sein des disciplines ou dans le cadre de démarches résolument interdisciplinaires.

2 Comme les coordinateurs l'annoncent dans une introduction très claire, l'approche privilégie est celle de l'histoire des sciences, et se propose d'analyser conjointement l'émergence des questions environnementales dans les sciences de la nature, la montée des préoccupations sociales et politiques qu'engendrent ces mêmes questions environnementales, et leur écho dans les disciplines de sciences humaines et sociales. Cet ancrage dans l'histoire des sciences imposait de remonter au XIX ${ }^{\mathrm{e}}$ siècle, à l'origine de ce que Wolf Feuerbahn (chapitre 1) désigne comme «les catégories de l'entendement écologique ", indépendamment des frontières entre disciplines de SHS. Les concepts de Milieu, précocement issu de la géographie hantée par le déterminisme, et d'Umvelt, proposé par certains philosophes allemands pour cerner les relations entre l'homme et la nature hors de toute démarche scientiste, sont mobilisés en vue d'expliquer l'émergence puis l'omniprésence actuelle de l'Environnement, un terme dont la polysémie traduit le foisonnement des acceptions diverses d'un concept souvent galvaudé et noyé dans la diversité des objets et des politiques environnementales, et appelle à une nécessaire clarification. 
3 L'objet majeur de l'ouvrage est l'évolution dans les quarante à cinquante dernières années des concepts, des pratiques, des courants et des structurations de neuf disciplines distinctes et identifiées de façon traditionnelle: anthropologie, histoire, philosophie, géographie, sociologie, critique littéraire, science politique, économie, droit. Ces disciplines sont étudiées comme autant de chapitres distincts, de 20 à 30 pages, confiés à des chercheurs engagés dans les débats que suscite l'environnement au sein de leur discipline.

4 Élise Demeulenaere (chapitre 2) propose en premier lieu une analyse argumentée et engagée des effets de l'irruption d'une anthropologie se consacrant les modalités d'usage et de connaissance la nature, au sein d'une discipline qui, longtemps partagée entre approches marxistes et structuralistes, avait comme ambition de rechercher l'invariant humain dans la diversité des cultures. Les débats conceptuels autour du dépassement du dualisme Nature/Culture, illustré par les travaux de Philippe Descola, le développement parallèle d'une ethno-écologie de terrain qui pratique la pluridisciplinarité avec les Sciences de la Nature, et l'implication de l'anthropologie dans les débats de l'écologie politique sont autant d'aspects de la naissance d'une anthropologie "au-delà de l'Anthropos », dont Élise Demeulenaere ne masque pas les difficultés d'insertion dans les institutions de recherche françaises, en particulier le CNRS.

5 Guillaume Blanc (chapitre 3) souligne la relative précocité avec laquelle l'Histoire a pu intégrer l'environnement parmi les «Territoires de l'historien ». De l'intérêt pour de nouveaux objets hybrides intégrant les interactions entre l'environnement et l'histoire humaine, tels que le climat, les forêts, les pollutions industrielles, etc., l'histoire environnementale en est venue à se définir comme un champ bien identifié de la recherche historique, avec des concepts, des méthodes et des débats qui lui sont propres. Guillaume Blanc nous propose un tableau précis de cette évolution dans différents pays ou parties du Monde, avant d'en souligner la convergence et l'internationalisation; il plaide pour une extension de l'histoire de l'environnement proprement dit à celle des articulations avec les sciences, les gouvernements et les sociétés, aux relations historiques avec les pouvoirs et les idées politiques.

6 Formulé sous forme d'une question (« l'écologie a-t-elle des implications morales?»), le titre du chapitre 4, consacré à la philosophie de l'environnement, dû à Catherine Larrère, en définit parfaitement l'objet. Il propose un panorama des relations entre écologie et éthique telles qu'elles ont été abordées depuis les années 1970, essentiellement dans la philosophie anglo-saxonne, avec quelques ouvertures sur les travaux français, parmi lesquels ceux de l'auteure du chapitre ont une place éminente. L'éthique environnementale, qui s'intéresse à la place de l'Homme dans la Nature, y apparaît clairement influencée par les évolutions de la science écologique, en même temps que par les rapports entre savoirs scientifiques et croyances dans nos sociétés contemporaines.

7 Christian A. Kull et Simon P. J. Waterbury (chapitre 5) analysent les rendez-vous manqués entre la géographie et l'écologie politique, alors même que la géographie a depuis longtemps contribué à la connaissance des relations entre l'Homme et la Nature, et devrait pouvoir jouer un rôle central dans l'analyse des questions environnementales actuelles. Les auteurs en recherchent les raisons dans les histoires récentes de la géographie anglo-saxonne et de la géographie française, reprenant pour l'essentiel les analyses développées dans le colloque Géographie, écologie, politique: un climat de 
changement (Orléans, 2012) et les thèses du Manifeste pour une géographie environnementale (D. Chartier et E. Rodary, dir., 2016), auxquels ils ont eux-mêmes contribué.

8 Pour Lionel Charles, Bernard Kalaora et Chloé Vlassopoulos (chapitre 6), la sociologie ne s'est engagé que tardivement et incomplètement dans une recherche institutionnelle sur l'environnement, malgré l'intérêt précoce de célèbres dissidents de la discipline pour la question environnementale. La sociologie rurale et la sociologie des risques ont été parmi les premières à aborder des thèmes à l'évidence environnementaux, mais en ordre dispersé et à l'écart du centre de la discipline, fidèle à un paradigme anthropocentré et aux traditions durkheimienne ou marxiste, et à des méthodes d'enquête quantitative peu adaptées à l'étude de l'environnement. Cette incomplétude sociologique n'a pas totalement empêché nombre de sociologues de s'engager dans les programmes pluridisciplinaires pilotés par le CNRS, en particulier le PEVS et ses suites, ou de répondre à une demande étatique croissante d'enquêtes ciblées sur l'acceptation des risques ou la perception des mesures environnementales. Mais la construction d'une sociologie de l'environnement organisée et fondée sur des concepts permettant de dépasser la parcellisation et l'individualisme des approches, reste en chantier.

9 L'éco-critique, conçue comme étude du rapport entre littérature et environnement, constitue selon Stéphanie Posthumus (chapitre 7), un domaine qui est né dans les années 1970 aux États-Unis, et bien établi depuis dans la littérature anglophone. Elle a en revanche bien du mal à s'imposer dans une critique littérature francophone plus centrée sur le texte et le formalisme, et rétive à une approche qui, comme pour d'autres thématiques comme les indigenous, post-colonial ou queer-studies, intègre une dimension éthique jugée trop spécifique de la culture américaine et éloignée de la culture française. Analysant les cadres théoriques et les méthodes de l'écocritique anglosaxonne, Stéphanie Posthumus plaide pour une approche éco-culturelle centrée sur la façon dont la littérature contribue à faire évoluer les rapports entre les sociétés et l'environnement.

10 Luc Sémal (chapitre 8) analyse la théorie politique verte, branche des sciences politiques qui s'attache à comprendre ce que l'irruption de la question environnementale depuis les années 1970 change, pourrait ou devrait changer au monde des idées et des pratiques politiques. L'émergence controversée des idées écologistes à l'époque du rapport Meadows, l'irruption, l'enracinement et la diversification de l'idéologie et des mouvements écologistes dans les sociétés démocratiques, les politiques environnementales développées à différentes échelles (mondiale, nationale, locale) face à une crise écologique de plus en plus patente, sont autant de sujets de la théorie politique verte. Selon Luc Sémal, cette théorie politique verte doit répondre à un double engagement, épistémologique et politique, faisant le lien entre réflexions de la recherche académique et positions militantes, au sein de la science politique proprement dite et de la communauté plus vaste des chercheurs engagés dans les humanités environnementales.

11 À Valérie Boisvert (chapitre 9) revient la tâche difficile de présenter les attitudes pour le moins contradictoires qui coexistent (plutôt mal que bien) au sein des sciences économiques face à l'environnement. Longtemps ignorées ou niées par la science économique mainstream, les « externalités » environnementales devraient pour les uns se soumettre à la régulation par le marché, opposée à l'intervention régulatrice des 
États dont d'autres s'efforcent de rationaliser l'action et de quantifier l'efficacité. À l'opposé, une économie écologique naît de la critique radicale de la croissance et des indicateurs monétaires tels que le PIB, et s'oriente vers la recherche d'indicateurs centrés sur les services écosystémiques et prenant en compte les impacts de l'activité économique sur l'environnement (l'empreinte écologique par exemple). Au risque de la dispersion et au prix d'une visibilité moindre, une partie des économistes choisit des approches plus sectorielles et pragmatiques qui ont l'avantage de faciliter les rapprochements avec les autres disciplines au sein des humanités environnementales.

Meryem Deffairi (chapitre 10) s'efforce à son tour de cerner les contours imprécis et mouvants du droit de l'environnement, une discipline à la fois très ancienne, quand il s'agissait de réglementer les eaux et forêts à l'époque de Colbert ou de protéger les populations contre les nuisances et pollutions à l'ère industrielle, plus récent quand il s'est agi de codifier la protection de la nature, qui doit faire face à une inflation des codes et réglementations depuis que l'environnement s'est installé au premier plan des préoccupations de nos sociétés. Mais l'environnement reste un objet de droit insaisissable, faute d'une définition et d'une qualification juridique commune, que Meryem Deffairi recherche vainement dans le nombre croissant de textes qui y font référence en droit français (Charte de l'Environnement), européen ou international, entre la définition classique de l'environnement comme patrimoine commun, la reconnaissance d'un droit à l'environnement nécessairement anthropocentré, et les tentatives encore timides de l'émergence d'un droit de l'environnement per se.

13 Au terme de ce panorama des disciplines, Gregory Quenet (chapitre 11) évoque l'émergence dans le monde académique anglo-saxon, germanique et scandinave, voire en Chine, de formes d'organisation, sous forme d'instituts ou de réseaux, d'une recherche pluridisciplinaire se revendiquant des humanités environnementales. Il examine les obstacles structurels qui s'opposent en France à un tel mouvement. Grégory Quenet définit les humanités environnementales comme une méta-discipline ayant pour ambition de fédérer les sciences humaines et sociales autour de concepts communs destinés à aborder de front la question du changement global à l'ère de l'Anthropocène, à toutes échelles et si possible en lien avec les sciences de la nature et de la planète. Il leur attribue le rôle d'insérer la matérialité du changement global dans le social et le culturel, d'en restituer l'historicité, et de repenser nos disciplines à partir de la figure d'un chercheur engagé pour l'environnement. Dans la continuité de ce chapitre, les coordinateurs de l'ouvrage proposent une conclusion qui évoque ce que nous pourrions qualifier de grandeur et misère de l'interdisciplinarité, entre les différentes sciences humaines et sociales certes, mais plus encore entre SHS et sciences de la nature et de la planète. Par-delà les déclarations d'intention et les incitations programmatiques, elle reste pour eux un effort dont rien ne garantit qu'il soit fécond, mais qui mérite d'être tenté.

14 La lecture de ces 270 pages, toujours denses, argumentées et nuancées, nécessite un effort mais cet effort est à recommander aux chercheurs engagés dans des études environnementales à partir d'une des disciplines de sciences humaines et sociales traitées dans l'ouvrage, soucieux de porter un regard réflexif et historique sur la façon dont leur discipline aborde l'environnement, et/ou d'élargir leurs perspectives à l'ensemble des humanités environnementales. Le centrage de l'ouvrage sur l'épistémologie et l'histoire des sciences offre une perspective complémentaire de celles du Guide des humanités environnementales, coordonné par Aurélie Choné, Isabelle 
Hajek et Philippe Hamman, dont les 53 notices apportent une découverte par thèmes des façons de penser la nature en sciences sociales, et d'autres ouvrages qui proposent un point de vue plus personnel des rapports entre environnement et sciences humaines et sociales. L'appel à des spécialistes et le traitement par discipline aurait pu être un facteur d'hétérogénéité, comme c'est fréquemment le cas dans les ouvrages collectifs, Il n'en est rien, les auteurs semblant avoir répondu à un cahier des charges précis : approche par l'histoire récente de la discipline, comparaison systématique entre les évolutions dans le monde anglo-saxon (et parfois européen) et la situation en France, sans éviter les blocages conceptuels et institutionnels qui lui sont propres. Le lecteur soucieux d'approfondir le cas d'une discipline ou d'une autre disposera d'une très abondante bibliographie (1060 références). Un ouvrage qui incite à un travail approfondi plutôt qu'à une lecture rapide, qui peut figurer en bonne place dans la bibliothèque des habitués de Développement Durable \& Territoires.

\section{AUTEUR}

\section{CLAUDE KERGOMARD}

Claude Kergomard est géographe et climatologue, professeur à l'Université des Sciences et Technologies de Lille jusqu'en 2003, puis professeur et directeur du Département de Géographie à l'Ecole normale supérieure de Paris, co-directeur du Centre d'Enseignement et de Recherches sur l'Environnement et la Société (2003-2013). 